\title{
Isolation Screening and Selection of Phosphate Solubilizing Fungi from Maize Rhizosphere
}

\author{
G. Amrutha*, V.P. Savalgi, K.S. Jagadeesh and N.S. Hebsur \\ Department of Agricultural Microbiology, College of Agriculture, Dharwad, University of \\ Agricultural Sciences, Dharwad, Karnataka, India \\ *Corresponding author
}

\section{A B S T R A C T}

\section{Keywords \\ P-solubilization, Phytohormones, Cellulase, Pikovskaya's agar \\ Article Info \\ Accepted: \\ 08 June 2018 \\ Available Online: \\ 10 July 2018}

Laboratory experiments were conducted to isolate screen and select the efficient P-solubilizing fungal isolates from maize rhizosphere in the districts of Northern Karnataka. $21 \mathrm{P}$-solubilizing fungal isolates were obtained from the soil samples and were screened for phosphorus solubilizing ability on Pikovskaya's agar and broth M-10(1) showed highest phosphorus solubilizing activity. Five efficient isolates were selected and assessed for other functional activities such as zinc and potassium solubilization, phytohormone production (IAA and $\mathrm{GA}_{3}$ ) and cellulolytic activity. All five isolates were able to solubilize zinc and produce phytohormones. Among the five isolates M-10(1) was highest in phytohormone production and DM-3(2) was the only isolate to produce cellulase enzyme.

\section{Introduction}

Phosphorus is a limiting nutrient in crop production. Therefore, it regulates the crop growth and yield to the greater extent thus its deficiency becomes an important chemical factor restricting plant growth in soils. Various factors can be responsible for phosphorus availability to crop plants. These include the form of native soil phosphorus, the type of phosphorus applied to the soil and reaction. As a result of strong adsorption of phosphate by iron and aluminum oxides, less than 1 per cent of soil phosphorus is available for plant uptake (Xavier et al., 2011). Therefore, primary approach in management of phosphorus is to scavenge the native or fixed phosphorus and also to minimize the fixation of applied phosphorus fertilizer. The low cost practice to achieve this objective is to inoculate soil with the phosphorus solubilizing microorganisms.

Microbial solubilization of inorganic phosphate occurs by various mechanisms, such as acidification, chelation, ion exchange reactions and polymeric substances formation. There are two ways in microbial phosphorus solubilization, by solubilization processes and from $\mathrm{P}$ accumulation in the microbial biomass. The phosphorus solubilizing microorganisms 
include fungi, bacteria and actinomycetes. Among these P-solubilizing microorganisms fungi have been reported to possess greater ability to solubilize insoluble phosphate than bacteria. Since phosphorus solubilizing fungi does not lose the phosphorus dissolving activity upon repeated sub culturing under laboratory conditions as the phosphorus solubilizing bacteria do (Sperber, 1958; Kucey, 1983) and they produce more acids than bacteria (Venkateswarlu et al., 1984). Moreover, fungi in soils are able to traverse long distances more easily than bacteria and hence, may be more important to phosphorus solubilization in soils (Kucey, 1983). Several soil fungi, particularly those belonging to the genera Penicillium and Aspergillus possess ability to bring insoluble soil phosphates into soluble forms by secreting weak organic acids such as formic, acetic, propionic, lactic, gluconic, fumaric and succinic acid.

\section{Materials and Methods}

The present study was conducted at Department of Agricultural Microbiology, University of Agricultural Sciences, Dharwad. Objectives of the present study were to isolate, screen and select the phosphorus solubilizing fungi from different locations of northern Karnataka (Belagavi, Haveri, Dharwad districts). The soil samples were collected from maize rhizosphere for isolation of $\mathrm{P}$ solubilizing fungi, covering three districts of Northern Karnataka and the geographical details of the study area are given in Table 1 .

\section{Isolation of P-solubilizing fungi from soil samples}

Isolation of P-solubilizing fungi was carried out by serial dilution and pour plate technique. Pikovskaya's agar amended with tri-calcium phosphate was used to isolate the $\mathrm{P}$ solubilizing fungi. The clear halo zone around the colonies was considered as positive result for P-solubilization.
Colony morphology of phosphorus solubilizing fungal isolates on solid media

The colour, type and shape of the phosphorus solubilizing fungal colonies were studied. The fungal isolates were grown on potato dextrose agar medium for one week at $28 \pm 2{ }^{\circ} \mathrm{C}$ and the colony characters were recorded.

Screening of phosphorus solubilizing fungal isolates (PSF) by zone of solubilization and inorganic phosphate $(\mathrm{Pi})$ released from tricalcium phosphate

The phosphorus solubilizing fungal isolates were screened for their phosphorus solubilizing ability and inorganic phosphate release. The quantitative assay (zone of solubilization) was carried out for all the 21 phosphorus solubilizing fungal isolates by spotting $10 \mu \mathrm{l}$ of spore suspension of the culture on sterile Pikovskaya's medium (Pikovskaya, 1948) and the zone of solubilization was calculated.

Inorganic phosphate $(\mathrm{Pi})$ released from tricalcium phosphate was estimated in pikovskaya's broth amended with tri-calcium phosphate. Cultures were grown in Pikovskaya's broth and the amount of $P i$ released in the broth was estimated at 5, 10 and 15 days after incubation in comparison with the un-inoculated control. The TCP broth cultures were spun at $10,000 \mathrm{rpm}$ for 10 minutes to separate the cells and insoluble phosphate. The available phosphorus content in the supernatant was estimated by phosphomolybdic blue colour method (Jackson, 1973).

\section{Selection and tentative identification of the phosphorus solubilizing fungal isolates}

Based on the solubilization index and inorganic phosphorus release by the fungal isolates, five morphologically different, compatible and efficient phosphorus 
solubilizing fungal isolates were selected and recoded for further experiment.

The isolates were tentatively identified up to generic level by observing the spore structure and arrangement in compound microscope using lactophenol blue.

\section{Phytohormone production}

The selected isolates were tested for production of phytohormones such as Indole 3-acetic acid and Gibberellic acid. The production of IAA was estimated by following the method given by Gordon and Paleg, (1957) and Gibberellic acid $\left(\mathrm{GA}_{3}\right)$ production was tested in Czapeck's broth containing 1 per cent glucose and peptone. The test organisms were inoculated in the broth. After 10 days of incubation the concentration of $\mathrm{GA}_{3}$ in culture broth was determined by spectrophotometric method using phosphomolybdic acid reagent (Deshmukh and Shinde, 2016).

\section{Potassium solubilization}

The selected PSF isolates were spotted on sterile Alexandrove's agar plates and incubated at $28 \pm 2{ }^{\circ} \mathrm{C}$ for 72 hours. The isolates having potassium solubilizing ability showed clear zone around the colony.

\section{Zinc solubilization}

The selected PSF isolates were spotted on the sterile liquid mineral salt medium specified by Saravan et al., (2003) and incubated at $28 \pm 2^{\circ} \mathrm{C}$ for 72 hours. The isolates having zinc solubilizing ability showed clear zone around the colony which indicated the solubilization of zinc in agar plates.

\section{Cellulolytic activity}

Fungal cultures were spotted on carboxy methyl cellulose agar media (CMC agar) and incubated at $28 \pm 2^{\circ} \mathrm{C}$ for 3 days. The plates were flooded with 0.1 per cent aqueous congo red and allowed for $30 \mathrm{~min}$, washed with $1 \mathrm{ml}$ $\mathrm{NaCl}$ (sodium chloride 1M) and allowed for $15 \mathrm{~min}$. the colonies that hydrolyzed cellulose showed clear zones against red colour of nonhydrolyzed medium (Sicuia et al., 2016).

\section{Results and Discussion}

Isolation and In vitro screening of phosphate solubilizing fungi (PSF)

A total of 47 rhizosphere soil samples were collected from three districts of Northern Karnataka (Haveri, Dharwad, Belagavi). The details such as location and geographical position of the site of soil sampling were taken (Table 1). Twenty one P-solubilizing fungal isolates were isolated from the collected soil samples (Table 2). The isolated fungal colonies were observed for colony morphology and the details are listed in Table 2. The isolates were assayed for in vitro phosphorus solubilization. The zone of solubilization, phosphate solubilization index and inorganic phosphate release by the isolates were assessed and the results are furnished in table 3.

Among all isolates M-10(1) showed maximum zone of solubilization and phosphate solubilization index $(1.0 \mathrm{~cm}$ and 3.0$)$ which was on par with M-9(5) (0.99 cm and 2.99) and BM-9(5) (0.99 cm and 2.99). However, the lowest was recorded by DM-2(2) $(0.1 \mathrm{~cm}$ and 2.1).

The inorganic phosphate $(\mathrm{Pi})$ release by the fungal isolates increased with incubation time and was highest at 15 days incubation time. The maximum $P i$ release was observed in M10(1) (10.17 ppm) followed by BM-9(5) (9.91 ppm) and M-9(5) (9.86 ppm) at $15^{\text {th }}$ day. The lowest $P i$ release was observed in DM-2(2) (3.44 ppm). The $P i$ release in the broth at 15 days of incubation ranged from 10.17 to 3.44 ppm (Table 3). 
Selection and tentative identification of the PSF isolates

Considering the phosphate solubilization and colony morphology, five morphologically different and biocompatible isolates with high phosphate solubilizing ability were selected for further study (M-10(1), M-9(5), BM-18(2), BM-9(5) and DM-3(2)). The selected PSF isolates were identified up to generic level based on their microscopic observation of spore structure and arrangement (Table 4).

\section{In vitro synthesis of IAA and $\mathrm{GA}_{3}$}

The production of IAA by PSF isolates varied from 5.96 to $7.84 \mu \mathrm{g}$ per $\mathrm{ml}$ of broth medium. Among all the isolates verified, M-10(1) produced high amount of IAA $(7.84 \mu \mathrm{g} / \mathrm{ml}$ of medium) followed by M-9(5) $(7.28 \mu \mathrm{g} / \mathrm{ml}$ of medium). Lowest amount of IAA was synthesized by BM-18(2) $(5.96 \mu \mathrm{g} / \mathrm{ml}$ of medium) (Table 4). However, the production of $\mathrm{GA}_{3}$ by PSF isolates varied from 2.31 to
$2.96 \mu \mathrm{g}$ per 25 mlof broth medium. Among all the isolates verified, M-10(1) produced highest amount of $\mathrm{GA}_{3}(2.96 \mu \mathrm{g} / 25 \mathrm{ml}$ of medium) followed by M-9(5) $(2.72 \mu \mathrm{g} / \mathrm{ml}$ of medium). Lowest amount of $\mathrm{GA}_{3}$ was synthesized by BM-18(2) $(2.31 \mu \mathrm{g} / 25 \mathrm{ml}$ of medium) (Table 4).

\section{Potassium and zinc solubilization}

Zinc and potassium solubilizing abilities of the PSF isolates were tested using $\mathrm{ZnO}$ mineral salt agar and Alexandrove's agar respectively. All the PSF isolates showed zinc solubilization, whereas none of them showed potassium solubilization (Table 4).

\section{Cellulolytic activity of PSF isolates}

All the selected PSF isolates were assessed for their cellulolytic activity on carboxy methyl cellulose agar (CMC) medium. Among all the isolates tested only DM-3(2) showed cellulose degradation (Table 4).

Fig.1 Inorganic phosphate $(P i)$ release by the phosphorus solubilizing fungal isolates in Pikovskaya's broth

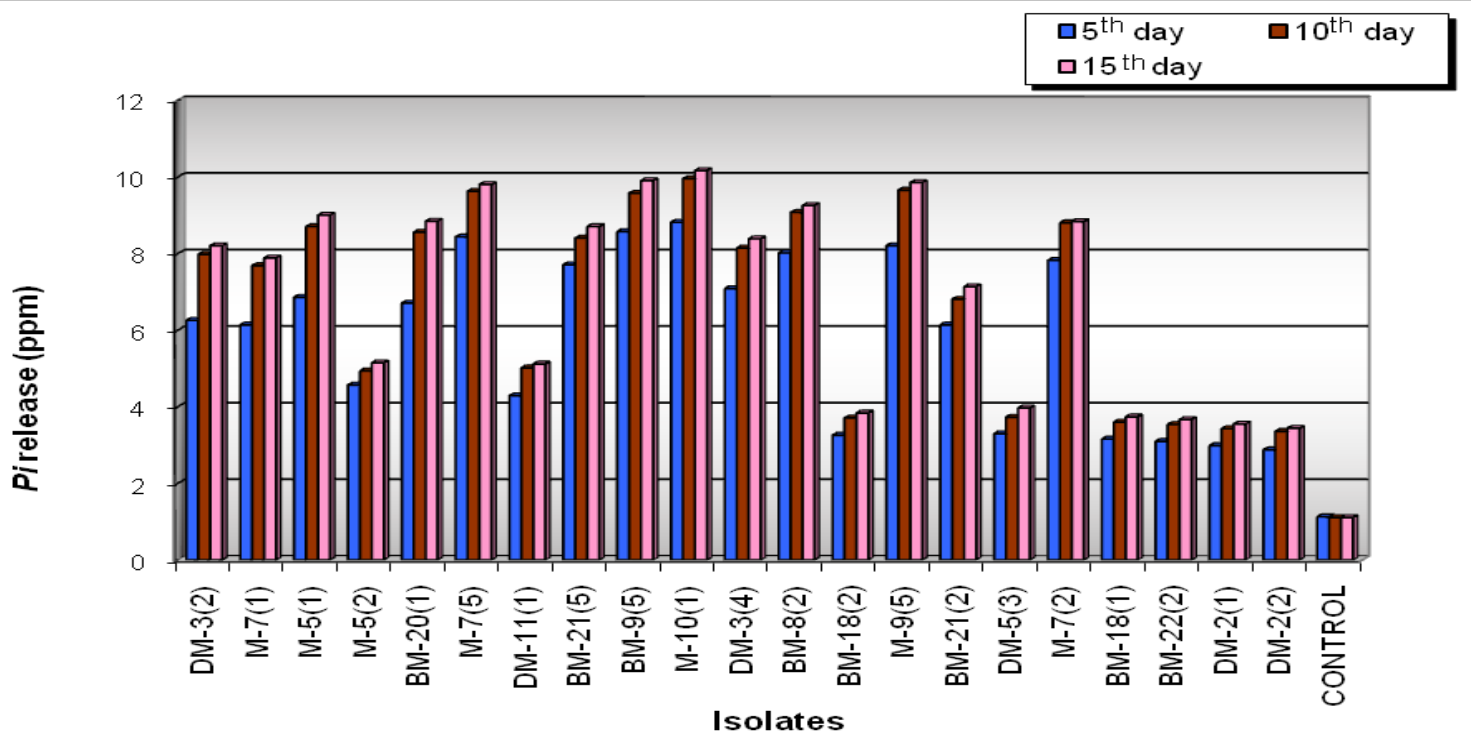


Table.1 Geographical positions of soil sampling sites

\begin{tabular}{|c|c|c|c|c|c|}
\hline $\begin{array}{l}\text { Sl. } \\
\text { No. }\end{array}$ & $\begin{array}{l}\text { Sample } \\
\text { number }\end{array}$ & Location & Latitude (N) & Longitude (E) & $\begin{array}{l}\text { Elevation } \\
\text { (m) }\end{array}$ \\
\hline \multicolumn{6}{|c|}{ Haveri } \\
\hline 01 & M-1 & Ranebennur & $14^{\circ} 37^{\prime} 20.7^{\prime \prime}$ & $75^{\circ} 36^{\prime} 29.1^{\prime \prime}$ & 595 \\
\hline $\mathbf{0 2}$ & M-2 & Hanumanamatti & $14^{\circ} 39^{\prime} 13.9^{\prime \prime}$ & $75^{\circ} 34^{\prime} 07.0^{\prime \prime}$ & 598 \\
\hline 03 & M-3 & Kakola & $14^{\circ} 41^{\prime} 06.5^{\prime \prime}$ & $75^{\circ} 32^{\prime} 21.5^{\prime \prime}$ & 606 \\
\hline 04 & M-4 & Motebennur & $14^{\circ} 43^{\prime} 38.3^{\prime \prime}$ & $75^{\circ} 28^{\prime} 14.2^{\prime \prime}$ & 595 \\
\hline 05 & M-5 & Haveri (Hanagal road) & $14^{\circ} 47^{\prime} 10.2^{\prime \prime}$ & $75^{\circ} 23^{\prime} 00.0^{\prime \prime}$ & 575 \\
\hline 06 & M-6 & Haveri & $14^{\circ} 49^{\prime} 44.4^{\prime \prime}$ & $75^{\circ} 21^{\prime} 50.0^{\prime \prime}$ & 561 \\
\hline 07 & M-7 & Punemanehalli & $14^{\circ} 52^{\prime} 53.6^{\prime \prime}$ & $75^{\circ} 18^{\prime} 32.7^{\prime \prime}$ & 559 \\
\hline 08 & M-8 & Bankapur & $14^{\circ} 55^{\prime} 44.3^{\prime \prime}$ & $75^{\circ} 16^{\prime} 06.7^{\prime \prime}$ & 565 \\
\hline 09 & M-9 & Bisnalli & $14^{\circ} 57^{\prime} 21.0^{\prime \prime}$ & $75^{\circ} 14^{\prime} 41.7^{\prime \prime}$ & 596 \\
\hline 10 & M-10 & Shiggaon & $15^{\circ} 00^{\prime} 22.8^{\prime \prime}$ & $75^{\circ} 12^{\prime} 05.1^{\prime \prime}$ & 647 \\
\hline 11 & M-11 & Kadahalli & $15^{\circ} 03^{\prime} 18.4^{\prime \prime}$ & $75^{\circ} 10^{\prime} 02.0^{\prime \prime}$ & 688 \\
\hline 12 & M-12 & Jigaluru & $15^{\circ} 05^{\prime} 40.2^{\prime \prime}$ & $75^{\circ} 09^{\prime} 08.7^{\prime \prime}$ & 661 \\
\hline 13 & M-13 & Varur & $15^{\circ} 11^{\prime} 59.9^{\prime \prime}$ & $75^{\circ} 08^{\prime} 11.2^{\prime \prime}$ & 631 \\
\hline \multicolumn{6}{|c|}{ Belagavi } \\
\hline 01 & BM-1 & Kittur & $15^{\circ} 40^{\prime} 55.9^{\prime \prime}$ & $74^{\circ} 54^{\prime} 58.9^{\prime \prime}$ & 682 \\
\hline 02 & BM-2 & Timmapur & $15^{\circ} 42^{\prime} 29.6^{\prime \prime}$ & $74^{\circ} 54^{\prime} 54.3^{\prime \prime}$ & 650 \\
\hline 03 & BM-3 & Shigihalli & $15^{\circ} 43^{\prime} 47.7^{\prime \prime}$ & $74^{\circ} 53^{\prime} 10.5^{\prime \prime}$ & 668 \\
\hline 04 & BM-4 & Dastikoppa & $15^{\circ} 48^{\prime} 19.6^{\prime \prime}$ & $74^{\circ} 51^{\prime} 57.4^{\prime \prime}$ & 657 \\
\hline 05 & BM-5 & M K Hubli & $15^{\circ} 50^{\prime} 17.5^{\prime \prime}$ & $74^{\circ} 52^{\prime} 33.4^{\prime \prime}$ & 676 \\
\hline 06 & BM-6 & Hirebagewadi & $15^{\circ} 56^{\prime} 24.1^{\prime \prime}$ & $74^{\circ} 57^{\prime} 17.3^{\prime \prime}$ & 682 \\
\hline 07 & BM-7 & Halaga & $15^{\circ} 54^{\prime} 32.6^{\prime \prime}$ & $74^{\circ} 49^{\prime} 54.3^{\prime \prime}$ & 745 \\
\hline 08 & BM-8 & Mutenatti & $15^{\circ} 54^{\prime} 06.3^{\prime \prime}$ & $74^{\circ} 47^{\prime} 08.8^{\prime \prime}$ & 761 \\
\hline 09 & BM-9 & Hallabavi & $15^{\circ} 55^{\prime} 33.0^{\prime \prime}$ & $74^{\circ} 46^{\prime} 55.9^{\prime \prime}$ & 760 \\
\hline 10 & BM-10 & Hattaragi & $15^{\circ} 56^{\prime} 32.0^{\prime \prime}$ & $74^{\circ} 47^{\prime} 56.1^{\prime \prime}$ & 736 \\
\hline 11 & BM-11 & Gotur & $15^{\circ} 56^{\prime} 46.1^{\prime \prime}$ & $74^{\circ} 48^{\prime} 07.6^{\prime \prime}$ & 748 \\
\hline 12 & BM-12 & Hukkeri & $16^{\circ} 00^{\prime} 36.1^{\prime \prime}$ & $74^{\circ} 49^{\prime} 39.3^{\prime \prime}$ & 553 \\
\hline 13 & BM-13 & Ghataprabha & $16^{\circ} 10^{\prime} 51.9^{\prime \prime}$ & $74^{\circ} 49^{\prime} 53.0^{\prime \prime}$ & 552 \\
\hline 14 & BM-14 & Arabhavi & $16^{\circ} 10^{\prime} 56.8^{\prime \prime}$ & $74^{\circ} 49^{\prime} 52.7^{\prime \prime}$ & 542 \\
\hline 15 & BM-15 & Gokak & $16^{\circ} 10^{\prime} 52.2^{\prime \prime}$ & $74^{\circ} 49^{\prime} 51.4^{\prime \prime}$ & 552 \\
\hline 16 & BM-16 & Kolavi & $16^{\circ} 12^{\prime} 41.7^{\prime \prime}$ & $74^{\circ} 46^{\prime} 21.0^{\prime \prime}$ & 561 \\
\hline 17 & BM-17 & Khanagaon & $16^{\circ} 14^{\prime} 43.1^{\prime \prime}$ & $74^{\circ} 41^{\prime} 33.5^{\prime \prime}$ & 621 \\
\hline 18 & BM-18 & Nesaragi & $16^{\circ} 14^{\prime} 20.1^{\prime \prime}$ & $74^{\circ} 37^{\prime} 01.7^{\prime \prime}$ & 671 \\
\hline 19 & BM-19 & Harugoppa & $16^{\circ} 09^{\prime} 21.8^{\prime \prime}$ & $74^{\circ} 32^{\prime} 56.7^{\prime \prime}$ & 675 \\
\hline 20 & BM-20 & Murgod & $16^{\circ} 05^{\prime} 30.4^{\prime \prime}$ & $74^{\circ} 31^{\prime} 06.5^{\prime \prime}$ & 686 \\
\hline 21 & BM-21 & Bailhongal & $15^{\circ} 57^{\prime} 31.4^{\prime \prime}$ & $74^{\circ} 30^{\prime} 50.6^{\prime \prime}$ & 743 \\
\hline 22 & BM-22 & Belavadi & $15^{\circ} 39^{\prime} 47.6^{\prime \prime}$ & $74^{\circ} 44^{\prime} 02.5^{\prime \prime}$ & 662 \\
\hline
\end{tabular}


Table.1 Contd.....

\begin{tabular}{|c|c|l|c|c|c|}
\hline $\begin{array}{c}\text { S1. } \\
\text { No. }\end{array}$ & $\begin{array}{c}\text { Sample } \\
\text { number }\end{array}$ & \multicolumn{1}{|c|}{ Location } & Latitude (N) & Longitude (E) & $\begin{array}{c}\text { Elevation } \\
(\mathrm{m})\end{array}$ \\
\hline & & \multicolumn{2}{|c|}{ Dharwad } & \\
\hline $\mathbf{0 1}$ & DM-1 & Kanavihonnapura & $15^{\circ} 40^{\prime} 56.1^{\prime \prime}$ & $74^{\circ} 54^{\prime} 58.2^{\prime \prime}$ & 680 \\
\hline $\mathbf{0 2}$ & DM-2 & Kanavihonnapura & $15^{\circ} 22^{\prime} 09.2^{\prime \prime}$ & $75^{\circ} 00^{\prime} 22.1^{\prime \prime}$ & 687 \\
\hline $\mathbf{0 3}$ & DM-3 & Jodahalli & $15^{\circ} 20^{\prime} 16.0^{\prime \prime}$ & $75^{\circ} 00^{\prime} 31.6^{\prime \prime}$ & 617 \\
\hline $\mathbf{0 4}$ & DM-4 & Hirehonnalli & $15^{\circ} 13^{\prime} 00.7^{\prime \prime}$ & $75^{\circ} 00^{\prime} 04.7^{\prime \prime}$ & 543 \\
\hline $\mathbf{0 5}$ & DM-5 & Harogera cross & $15^{\circ} 13^{\prime} 19.4^{\prime \prime}$ & $75^{\circ} 01^{\prime} 00.4^{\prime \prime}$ & 576 \\
\hline $\mathbf{0 6}$ & DM-6 & Kamadhenu cross & $15^{\circ} 14^{\prime} 00.3^{\prime \prime}$ & $75^{\circ} 01^{\prime} 35.4^{\prime \prime}$ & 581 \\
\hline $\mathbf{0 7}$ & DM-7 & Ukkinakere & $15^{\circ} 15^{\prime} 08.0^{\prime \prime}$ & $75^{\circ} 02^{\prime} 25.0^{\prime \prime}$ & 574 \\
\hline $\mathbf{0 8}$ & DM-8 & Kusugal & $15^{\circ} 23^{\prime} 19.6^{\prime \prime}$ & $75^{\circ} 12^{\prime} 24.8^{\prime \prime}$ & 642 \\
\hline $\mathbf{0 9}$ & DM-9 & Behatti & $15^{\circ} 27^{\prime} 12.5^{\prime \prime}$ & $75^{\circ} 14^{\prime} 33.1^{\prime \prime}$ & 610 \\
\hline $\mathbf{1 0}$ & DM-10 & Sulla & $15^{\circ} 27^{\prime} 04.9^{\prime \prime}$ & $75^{\circ} 11^{\prime} 56.4^{\prime \prime}$ & 619 \\
\hline $\mathbf{1 1}$ & DM-11 & Sulla & $15^{\circ} 27^{\prime} 12.9^{\prime \prime}$ & $75^{\circ} 10^{\prime} 41.2^{\prime \prime}$ & 640 \\
\hline $\mathbf{1 2}$ & DM-12 & Somanahalli & $15^{\circ} 29^{\prime} 22.6^{\prime \prime}$ & $75^{\circ} 04^{\prime} 42.0^{\prime \prime}$ & 660 \\
\hline
\end{tabular}

Table.2 Colony morphology of phosphorus solubilizing fungal (PSF) isolates on potato dextrose agar

\begin{tabular}{|c|c|c|c|c|}
\hline Sl. No. & Isolates & Colony colour & Texture & Margin \\
\hline 01 & DM-5(3) & Greenish white & Flat & Round, even \\
\hline 02 & BM-18(1) & Black & Cottony & Round, even \\
\hline 03 & DM-2(1) & Black & Flat & Round, even \\
\hline 04 & DM-2(2) & White colony brown center & Flat, powdery & Round, uneven \\
\hline 05 & BM-18(2) & White colony brown center & Flat, powdery & Round, uneven \\
\hline 06 & $\mathrm{BM}-22(2)$ & Black & Flat & Round, even \\
\hline 07 & M-7(2) & Bottle green with white mycelia & Raised & Round, uneven \\
\hline 08 & M-5(1) & Black & Flat & Round, even \\
\hline 09 & DM-3(4) & Bottle green with white mycelia & Raised & Round, uneven \\
\hline 10 & BM-9(5) & Black & Flat & Round, even \\
\hline 11 & M-10(1) & Black & Flat & Round, even \\
\hline 12 & BM-20(1) & Black & Flat & Round, even \\
\hline 13 & DM-3(2) & White colony brown center & Flat, powdery & Round, uneven \\
\hline 14 & DM-11(1) & Black & Flat & Round, even \\
\hline 15 & M-9(5) & Orange & Flat, powdery & Round, even \\
\hline 16 & M-7(1) & Black & Flat & Round, even \\
\hline 17 & BM-21(5) & Black & Flat & Round, even \\
\hline 18 & M-7(5) & Black & Flat & Round, even \\
\hline 19 & M-5(2) & Black & Flat & Round, even \\
\hline 20 & BM-21(2) & Black & Flat & Round, even \\
\hline 21 & BM-8(2) & Black & Flat & Round, even \\
\hline
\end{tabular}


Table.3 Zone of solubilization and inorganic phosphate $(\mathrm{Pi})$ release by the phosphorus solubilizing fungal isolates on Pikovskaya's agar and broth

\begin{tabular}{|c|c|c|c|c|c|c|}
\hline \multirow{2}{*}{$\begin{array}{l}\text { Sl. } \\
\text { No. }\end{array}$} & \multirow[t]{2}{*}{ Isolates } & \multirow{2}{*}{$\begin{array}{l}\text { Zone of } \\
\text { solubilization } \\
(\mathrm{cm})\end{array}$} & \multirow{2}{*}{$\begin{array}{l}\text { Solubilization } \\
\text { index }\end{array}$} & \multicolumn{3}{|c|}{$P i$ release $(\mathrm{ppm})$} \\
\hline & & & & $5^{\text {th }}$ day & $10^{\text {th }}$ day & $15^{\text {th }}$ day \\
\hline 01 & DM-5(3) & 0.41 & 2.41 & 3.30 & 3.73 & 3.97 \\
\hline 02 & BM-18(1) & 0.26 & 2.26 & 3.26 & 3.71 & 3.84 \\
\hline 03 & DM-2(1) & 0.14 & 2.14 & 2.99 & 3.43 & 3.55 \\
\hline 04 & DM-2(2) & 0.10 & 2.10 & 2.88 & 3.36 & 3.44 \\
\hline 05 & BM-18(2) & 0.40 & 2.40 & 3.26 & 3.71 & 3.84 \\
\hline 06 & $\mathrm{BM}-22(2)$ & 0.12 & 2.12 & 3.10 & 3.54 & 3.67 \\
\hline 07 & $M-7(2)$ & 0.81 & 2.81 & 7.83 & 8.81 & 8.84 \\
\hline 08 & $M-5(1)$ & 0.93 & 2.93 & 6.86 & 8.71 & 9.01 \\
\hline 09 & DM-3(4) & 0.79 & 2.79 & 7.09 & 8.15 & 8.39 \\
\hline 10 & $\mathrm{BM}-9(5)$ & 0.99 & 2.99 & 8.58 & 9.58 & 9.91 \\
\hline 11 & M-10(1) & 1.00 & 3.00 & 8.82 & 9.96 & 10.17 \\
\hline 12 & BM-20(1) & 0.92 & 2.92 & 6.71 & 8.56 & 8.85 \\
\hline 13 & DM-3(2) & 0.88 & 2.88 & 6.26 & 7.98 & 8.21 \\
\hline 14 & DM-11(1) & 0.61 & 2.61 & 4.29 & 5.02 & 5.12 \\
\hline 15 & M-9(5) & 0.99 & 2.99 & 8.21 & 9.66 & 9.86 \\
\hline 16 & $\mathrm{M}-7(1)$ & 0.81 & 2.81 & 6.14 & 7.69 & 7.89 \\
\hline 17 & BM-21(5) & 0.92 & 2.92 & 7.71 & 8.41 & 8.71 \\
\hline 18 & $M-5(2)$ & 0.67 & 2.67 & 4.57 & 4.94 & 5.15 \\
\hline 19 & $M-7(5)$ & 0.97 & 2.97 & 8.44 & 9.63 & 9.81 \\
\hline 20 & BM-21(2) & 0.74 & 2.74 & 6.14 & 6.81 & 7.14 \\
\hline 21 & $\mathrm{BM}-8(2)$ & 0.86 & 2.86 & 8.02 & 9.08 & 9.26 \\
\hline \multicolumn{2}{|c|}{ S. Em. \pm} & 0.035 & 0.035 & 0.035 & 0.036 & 0.030 \\
\hline \multicolumn{2}{|c|}{ C.D. at $1 \%$} & 0.141 & 0.141 & 0.140 & 0.145 & 0.121 \\
\hline
\end{tabular}


Table.4 Identification of selected PSF isolates up to generic level based on microscopic observation and their functional characterization

\begin{tabular}{|c|c|c|c|c|c|c|c|c|}
\hline Sl. No. & Isolate no. & $\begin{array}{c}\text { Probable } \\
\text { Genus }\end{array}$ & Spore character & $\begin{array}{c}\text { Potassium } \\
\text { solubilization }\end{array}$ & $\begin{array}{c}\text { Zinc } \\
\text { solubilization }\end{array}$ & $\begin{array}{c}\text { IAA } \\
(\mu \mathrm{g} / \mathrm{ml})\end{array}$ & $\begin{array}{c}\mathrm{GA} \\
(\mu \mathrm{g} / 25 \mathrm{ml})\end{array}$ & $\begin{array}{c}\text { Cellulolytic } \\
\text { activity }\end{array}$ \\
\hline 1 & M-10(1) & Aspergillus sp. & $\begin{array}{l}\text { Conidiophore terminated by } \\
\text { swollen vesicle bearing flask } \\
\text { shaped sterigmata which give rise } \\
\text { to chain of conidia }\end{array}$ & - & + & 7.84 & 2.96 & - \\
\hline 2 & $M-9(5)$ & Penicillium sp. & $\begin{array}{l}\text { Conidiophore terminated by } \\
\text { cluster of flask shaped sterigmata } \\
\text { which give rise to chain of conidia } \\
\text { which give finger like appearance }\end{array}$ & - & + & 7.28 & 2.72 & - \\
\hline 3 & BM-18(2) & Penicillium sp. & $\begin{array}{l}\text { Conidiophore terminated by } \\
\text { cluster of flask shaped sterigmata } \\
\text { which give rise to chain of conidia } \\
\text { which give finger like appearance }\end{array}$ & - & + & 5.96 & 2.31 & - \\
\hline 4 & BM-9(5) & Aspergillus sp. & $\begin{array}{l}\text { Conidiophore terminated by } \\
\text { swollen vesicle bearing flask } \\
\text { shaped sterigmata which give rise } \\
\text { to chain of conidia }\end{array}$ & - & + & 6.93 & 2.63 & - \\
\hline 5 & DM-3(2) & Unidentified & - & - & + & 6.52 & 2.44 & + \\
\hline
\end{tabular}


Plate.1 Phosphate solubilization by the fungal isolates on Pikovskaya's agar

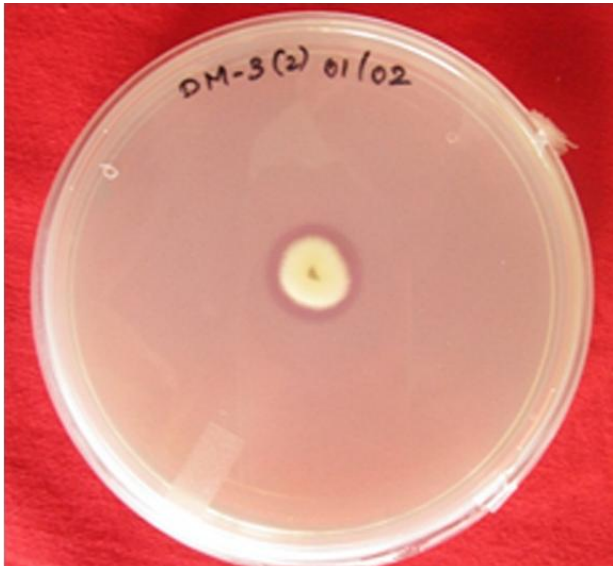

DM-3(2)

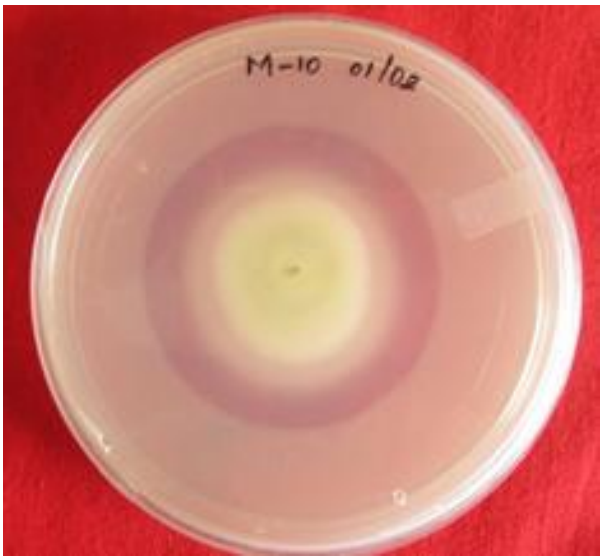

M-10(1)

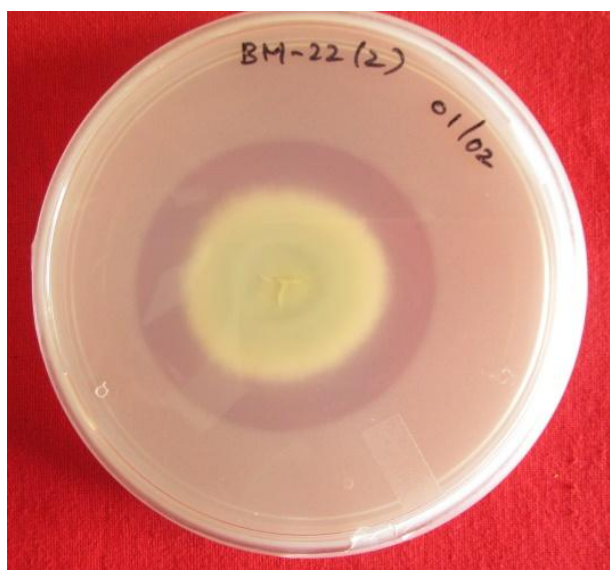

BH-22(2)

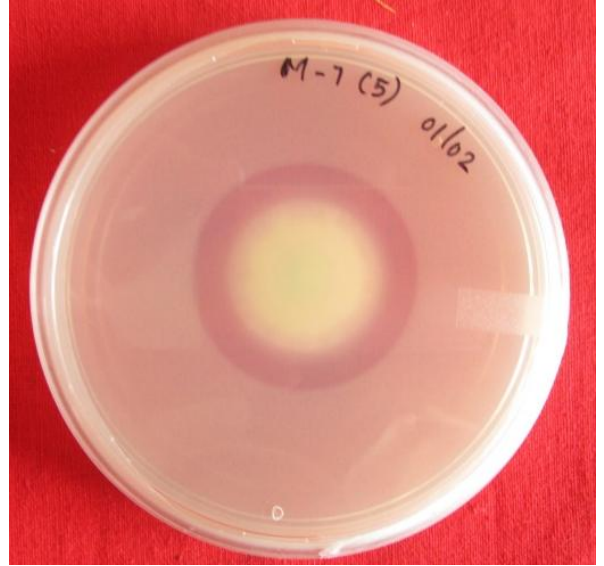

M-7(5)

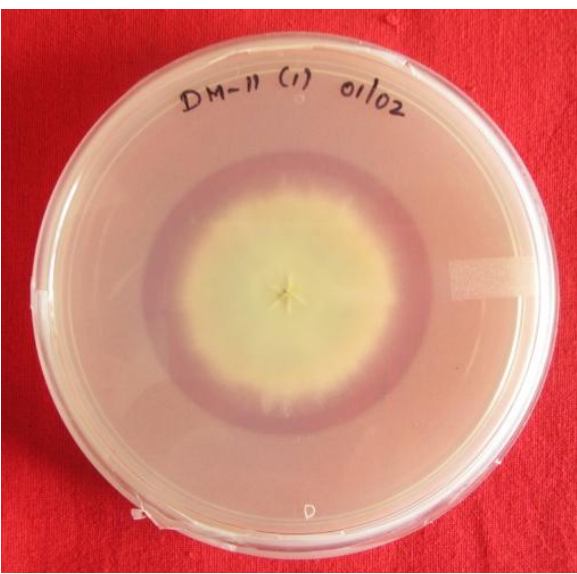

DM-11(1)

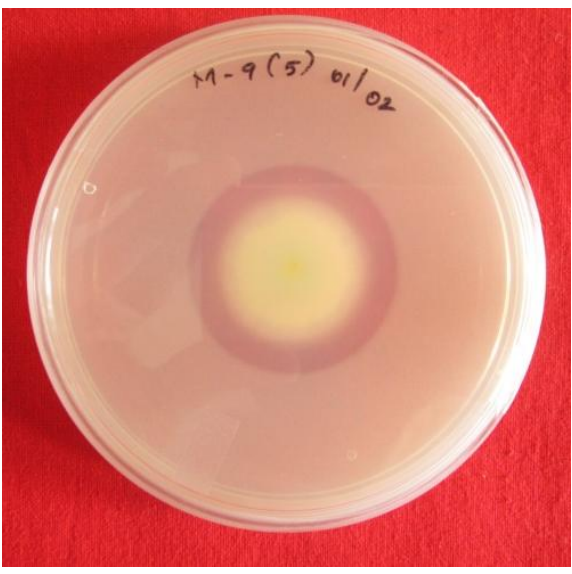

M-9(5) 
The soil samples were collected initially from rhizosphere of maize plant from three districts of Northern Karnataka (Haveri, Dharwad, Belagavi) for isolation of PSF. In this study, out of 47 samples $21 \mathrm{P}$ - solubilizing fungal isolates were isolated. The typical colony characters of the isolated PSF that appeared on Pikovskaya's agar ranged from flat powdery to raised and the colour of the colonies were green, black, brown, orange and white. The margin of the colony was either even or uneven. The isolated PSF were purified and maintained. Among the 21 PSF isolates M-10(1) showed maximum zone of solubilization, phosphate solubilization index and $P i$ release in Pikovskaya's agar and broth respectively followed by M-9(5) and BM-9(5) indicating that they exhibit strong mechanisms for solubilization of phosphate than other isolates. The variation in $\mathrm{P}$ solubilization could be due to the difference in the amount and type of the organic acids secreted which directly influence the $\mathrm{P}$ solubilization (Fig. 1).

Five morphologically different and efficient P-solubilizing fungal isolates were selected and tentatively identified based on their colony morphology and spore structure. Two isolates were identified as Aspergillus sp. and two isolates as Penicilluim sp. The selected Psolubilizing isolates were assessed for functional activities such as zinc and potassium solubilization, production of growth promoting hormones (IAA and $\mathrm{GA}_{3}$ ). All the isolates were able to solubilize zinc and produce growth promoting hormones such as IAA and $\mathrm{GA}_{3}$.Among the isolates, M10(1) was found to produce maximum amount of IAA and $\mathrm{GA}_{3}$ which was 7.84 $\mu \mathrm{g} / \mathrm{ml}$ and $2.96 \mu \mathrm{g} / \mathrm{ml}$ respectively. This property of the PSF isolates describes the importance of these microorganisms in plant growth which can in turn result in higher yield.
Cellulolytic activity is one of the very important traits for the better survival and ecological fitness of the organism in the rhizosphere (Boer et al., 2005). Hence the ability of P-solubilizing fungal isolates for cellulose degradation was tested on CMC agar medium. Cellulolytic activity of the selected 5 PSF isolates was tested on CMC agar medium. DM-3(2) showed positive result for cellulose hydrolysis. The cellulose degradation is due to the production of cellulase enzyme by the isolate which convert complex sugar cellulose into simple sugars.

\section{References}

Boer, W. D., Folman, L. B., Richard, C. S. and Lynne, B., 2005, Living in a fungal world: impact of fungi on soil bacterial niche development. FEMS Microbiol. Rev., 29(4): 795-811.

Deshmukh, P.D. and Shinde, S.Y., 2016, Gibberellic acid production by some fungi associated with Cajanus cajan Linn. Int. J. Sci. Info., 1(5): 279-283.

Gorden, S. A. and Paleg, L. G., 1957, Quantitative measurement of indole acetic Acid.Plant Physiol., 10: 37-48.

Jackson, M. L., 1973, Soil Chemical Analysis, Prentice Hall of India Private Limited, New Delhi, 38-82.

Kucey, R. M. N., 1983, Phosphate solubilizing bacteria and fungi in various cultivated and virgin Alberta soils. Canadian J. Soil Sci., 63: 671678.

Pikovskaya, R. L., 1948, Mobilization of phosphorus in soil in connection with vital activity of some microbial species. Microbiologiya, 17: 362-370.

Saravan, V. S., Subramonium, S. R. and Raj, S. A., 2003, Assessing in vitro solubilization of different zinc solubilizing bacterial isolates. Brazil J. Microbiol., 34: 121-125.

Sicuia, O., Grosu, I., Constantinescu, F., 
Voaides, C. and Petruta, C., 2016, Enzymatic and genetic variability in Bacillus sp. strains with plant beneficial qualities. Bull. No. 73 UASVM Agri., pp. 66-68.

Sperber, J. I., 1958, The incidence of apatitesolubilizing organisms in the rhizosphere and soil. Australian J. Agril. Res., 9: 778-781.

Venkateswarlu, B., Rao, A.V., Raina, P. and Ahmad, N., 1984, Evaluation of phosphorus solubilization by microorganisms isolated from arid soil. J. Indian Soc. Soil Sci., 32: 273277.

Xavier, F. A. S., Almeida, E. F., Cardoso, I. M. and de Mendonça, E. S., 2011, Soil phosphorus distribution in sequentially extracted fractions in tropical agroecosystems in the Atlantic forest biome, southeastern Brazil. Nutr.Cycl.Agroecosyst., 89: 31-44.

\section{How to cite this article:}

Amrutha, G., V.P. Savalgi, K.S. Jagadeesh and Hebsur, N.S. 2018. Isolation Screening and Selection of Phosphate Solubilizing Fungi from Maize Rhizosphere. Int.J.Curr.Microbiol.App.Sci. 7(07): 988-998. doi: https://doi.org/10.20546/ijcmas.2018.707.120 